\title{
Preclinical Evaluation of Antitumor Efficacy of a New Radiopharmaceutical Based on Thermoresponsive Carrier and Samarium-153
}

\author{
Petriev V.M. ${ }^{1}{ }^{1,2}$, Tishchenko V.K. ${ }^{1}$, Smoryzanova O.A. ${ }^{1}$, Bol'bit N.M. ${ }^{3}$, \\ Duflot V.R. ${ }^{3}$, Morozova N.B. ${ }^{4}$, and Yakubovskaya R.l.4 \\ ${ }^{1}$ Tsyb Medical Radiological Research Centre, Obninsk, Russia \\ ${ }^{2}$ National Research Nuclear University MEPhl (Moscow Engineering Physics Institute), \\ Kashirskoe shosse 31, Moscow, 115409, Russia \\ 3JSC «Karpov Institute of Physical Chemistry», Obninsk, Russia \\ ${ }^{4}$ Moscow Hertsen Research Institute of Oncology, Moscow, Russia
}

Corresponding Author:

Vasily M. Petriev

petriev@mrrc.obninsk.ru

Received: 17 January 2018

Accepted: 25 March 2018

Published: 17 April 2018

Publishing services provided by

Knowledge

(c) Petriev V.M. et al. This article is distributed under the terms of the Creative Commons

Attribution License, which permits unrestricted use and redistribution provided that the original author and source are credited.

Selection and Peer-review under the responsibility of the PhysBioSymp17 Conference Committee.

\section{Abstract}

This work is devoted to studying the in vivo antitumor efficacy of the new injection radiopharmaceutical based on thermoresponsive polymer and $\beta^{-}$-emitting radionuclide samarium-153 ( ${ }^{153} \mathrm{Sm}$-KARP-CheM).

The study of in vivo antitumor efficacy was performed using mice $\mathrm{F}_{1}$ and $\mathrm{C}_{57} \mathrm{Bl} / 6$ with transplanted subcutaneously sarcoma $S 37$ and melanoma B16, respectively. The animals received single intratumoral bolus injections of $37 \mathrm{MBq}(1 \mathrm{mCi})$, or $18.5 \mathrm{MBq}$ (0.5 mCi) of ${ }^{153} \mathrm{Sm}$-KARP-CheM, or saline in a volume $0.1 \mathrm{ml}$. The efficacy of antitumor treatment was evaluated using tumor growth inhibition index (TGI, \%) and increase of average life span (ILS, \%).

The most meaningful therapeutic efficacy after intratumoral injection of ${ }^{153} \mathrm{Sm}$-KARPCheM was observed in melanoma-bearing mice $\mathrm{C}_{57} \mathrm{Bl} / 6$. The highest values of TGI for melanoma B16 were $79.5 \%$ and $79.6 \%$ after treatment with $18.5 \mathrm{MBq}$ or 37 $\mathrm{MBq}$, respectively. An increase of average life span by $17.1 \%$ was found in group of melanoma-bearing mice treated with $37 \mathrm{MBq}$ of ${ }^{153} \mathrm{Sm}$-KARP-CheM only. Tumor growth inhibition of sarcoma $\mathrm{S}_{37}$ was slightly lower as compared with melanoma B16: $62.5 \%$ and $59.0 \%$ in $37 \mathrm{MBq}$ and $18.5 \mathrm{MBq}$ groups, respectively. ${ }^{153} \mathrm{Sm}$-KARP-CheM didn't increase average life span of treated animals. In conclusion, ${ }^{153} \mathrm{Sm}-\mathrm{KARP}-\mathrm{CheM}$ seems to be effective radiopharmaceutical for local tumor radiotherapy.

Keywords: thermoresponsive polymer, samarium-153, radionuclide therapy of cancer, sarcoma S37, melanoma B16, antitumor efficacy. 


\section{Introduction}

Nowadays the search of new approaches for cancer therapy is still continued. Local injectable radiotherapy is one of the promising method of solid tumors treatment. An intratumoral administration of labeled compound allows for depositing high radiation doses directly in the application site and keeping the whole-bode doses low.

Radiolabeled thermoresponsive, sometimes also called thermosensitive polymers attract much attention due to their cloud-point temperature values close to human body temperature $[1,2]$. In case the environment is heated above $37^{\circ} \mathrm{C}$, the polymer chains hydrate, become more hydrophobic, and finally collapse. It immediately leads to a high amount of radioactivity at the desired site of action, and avoids systemic adverse effects. Futhermore, in intratumoral radionuclide therapy the radionuclide does not need to be internalized by tumor cells to achieve efficacy, because the radioactive emissions can kill tumor cells from a distance.

One of the well-known and widely studied polymer is poly( $\mathrm{N}$-isopropylacrylamide) (PNIPAM) because of its well defined structure and property specially its temperature response is closed to human body [3]. It is not cytotoxic for any of human cell lines even at high concentration, and its excretion occurred via urine and faeces $[4,5]$.

Samarium-153 $\left({ }^{153} \mathrm{Sm}\right)$ is an appropriate radionuclide for radiotherapy of cancer. ${ }^{153} \mathrm{Sm}$ emits beta particles with maximum energy of $0.81 \mathrm{MeV}$, mean energy of 0.58 $\mathrm{MeV}$. The mean range of energy deposition is $0.6 \mathrm{~mm}$, while the maximum range is $2.5 \mathrm{~mm}$. The physical half-life of ${ }^{153} \mathrm{Sm}$ is 46.3 hours (1.9 days). It also emits a $103-\mathrm{keV}$ gamma radiation, which allows imaging of biodistribution of labeled compound with conventional gamma-cameras.

The aim of this study was to evaluate an antitumor efficacy of a new designed thermoresponsive system based on the copolymers of $\mathrm{N}$-isopropylmethacrylamide with allylamine labeled with ${ }^{153} \mathrm{Sm}$ for local radiotherapy.

\section{Methods and materials}

The in vivo antitumor efficacy of ${ }^{153} \mathrm{Sm}$-CARP-CheM was assessed using $\mathrm{C}_{57} \mathrm{Bl} / 6$ mice with melanoma B16 xenograft tumors and F1 mice with sarcoma S-37 xenograft tumors. When the tumor volumes reached $70-90 \mathrm{~mm}^{3}, 30 \mathrm{C} 57 \mathrm{Bl} / 6$ mice were randomized into 3 groups, and 30 F1 mice were also randomly separated into 3 groups. Each groups of animals were intratumoral injected with $37 \mathrm{MBq}(1 \mathrm{mCi})$, or $18.5 \mathrm{MBq}$ (0.5 mCi) of ${ }^{153} \mathrm{Sm}$-CARP-CheM, or saline in a volume $0.1 \mathrm{ml}$. Tumor size was determined 
twice a week by measuring the length, width, and depth of the tumor using a caliper. Tumor volume was calculated as follows: tumor volume $=$ (length $\times$ width $\times$ depth $) \times$ $\pi / 6$.

The efficacy of antitumor treatment was evaluated using tumor growth inhibition index (TGI, \%) and increase of average life span (ILS, \%). TGI was defined as:

$$
T G I=\left(1-\frac{\text { Mean volume of treated tumors }}{\text { Mean volume of control tumors }}\right) \times 100 \% \text {. }
$$

ILS was defined as:

$$
I L S=\left(1-\frac{\text { Average life span of treated animals }}{\text { Average life span of control animals }}\right) \times 100 \% .
$$

The results of mean tumor volume and average life span for each group of mice were expressed as mean value and standard error of the mean $(M \pm m)$. Student's $t$ test was used to analyze data throughout all studies between groups, and $p<0.05$ was considered statistically significant.

\section{Results}

Antitumor efficacy of ${ }^{153} \mathrm{Sm}$-CARP-CheM was assessed in tumor-bearing mice with different tumor histogenesis: melanoma B16 and sarcoma S-37. According to the results, intratumoral injection of ${ }^{153} \mathrm{Sm}$-CARP-CheM considerably inhibited tumor growth of melanoma B16 and sarcoma S-37. The most significant effect was observed in melanoma B16 tumor-bearing mice: intratumoral injection of ${ }^{153} \mathrm{Sm}$-CARP-CheM delayed tumor growth as compared with control group at 4 days after treatment (Fig. 1). There were no significant differences between $37 \mathrm{MBq}$ and 18.5 MBq groups. It was evident that the tumor volume for control mice increased 32 -fold to $3680.1 \mathrm{~mm}^{3}$ while the tumor volume of mice treated with $37 \mathrm{MBq}$ or $18.5 \mathrm{MBq}$ of ${ }^{153} \mathrm{Sm}$-CARP-CheM increased 15 -fold only (Fig. 1). TGI index varied from $55.4 \%$ to $79.6 \%$ in $37 \mathrm{MBq}$ treated group, and from $55.3 \%$ to $79.5 \%$ in $18.5 \mathrm{MBq}$ treated group of melanoma-bearing mice (Table 1).

In mice $\mathrm{F}_{1}$ with subcutaneous transplanted sarcoma S-37 significant tumor growth inhibition was revealed only at 11-14 days after treatment (Figure 1, Table 1). The tumor volume increased 12 -fold and 23 -fold throughout the study in $37 \mathrm{MBq}$ and $18.5 \mathrm{MBq}$ treated groups, respectively, and 36-fold in control group. In $18.5 \mathrm{MBq}$ group of mice the highest TGI index (62.5\%) was observed at 14 day after ${ }^{153} \mathrm{Sm}$-CARP-CheM injection, whereas in $37 \mathrm{MBq}$ group TGI index was reached the highest value (59.0\%) by the end of the study. 


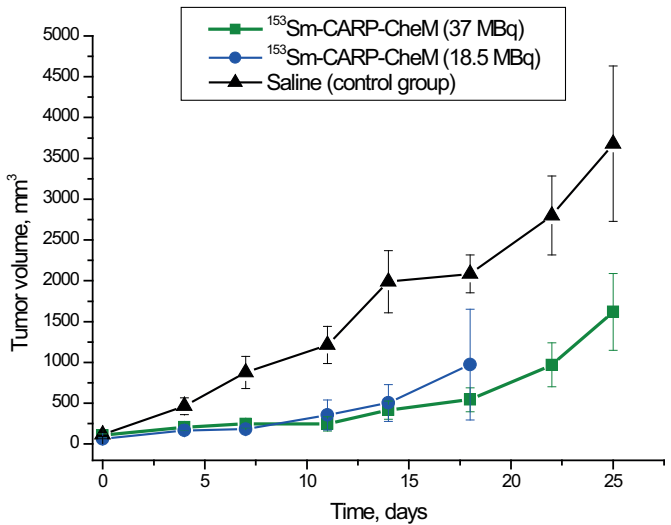

A

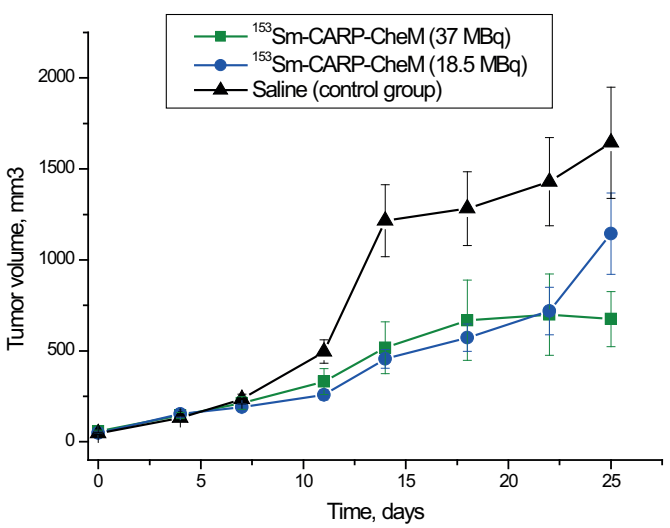

B

Figure 1: Mean tumor volume after intratumoral administration of $37 \mathrm{MBq}$ and $18.5 \mathrm{MBq}$ of ${ }^{153} \mathrm{Sm}$-CARPCheM, or saline: A - mice $\mathrm{C}_{57} \mathrm{Bl} / 6$ with subcutaneous transplanted melanoma B16; B - mice F1 with subcutaneous transplanted sarcoma S-37.

TABLE 1: Antitumor efficacy of ${ }^{153} \mathrm{Sm}$-CARP-Chem.

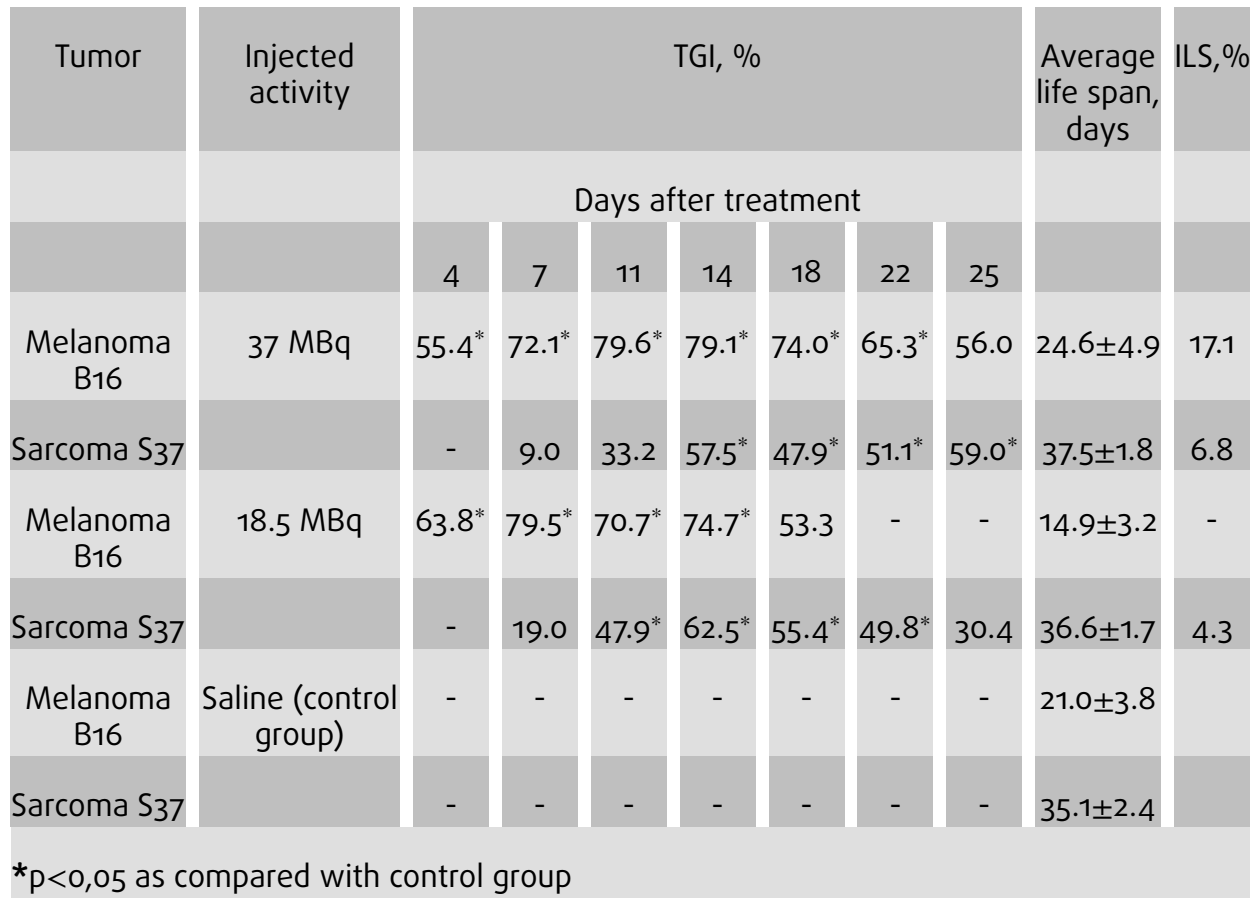

Average life span of melanoma B16 bearing mice treated with $37 \mathrm{MBq}$ of ${ }^{153} \mathrm{Sm}$ CARP-CheM was 24.6 days versus 21.0 days in control group ( $p>0.5$ ), and ILS was $17.1 \%$. In contrast, average life span in $18.5 \mathrm{MBq}$ group decreased by $29 \%$ as compared with control group and didn't exceed 14.9 days (Table 1 ).

In mice with sarcoma S-37 average life span was 37.5 days and 36.6 days in $37 \mathrm{MBq}$ and 18.5 MBq groups, respectively. There were no significant differences between the average life span in both treated groups of mice F1 with sarcoma S-37 and control 
group. The values of ILS were only $6.8 \%$ and $4.3 \%$ in groups that received $37 \mathrm{MBq}$ or 18.5 MBq of ${ }^{153} \mathrm{Sm}$-CARP-CheM, respectively (Table 1 ).

\section{Conclusion}

Intratumoral injection of ${ }^{153} \mathrm{Sm}$-CARP-CheM considerably inhibited tumor growth of melanoma B16 and sarcoma S-37. The most significant effect was observed in melanoma B16 tumor-bearing mice: TGI index reached $79.6 \%$ and $79.5 \%$ in $37 \mathrm{MBq}$ and 18.5 MBq treated groups, respectively. Tumor growth inhibition of sarcoma S37 was slightly lower as compared with melanoma B16: 62.5\% and 59.0\% in $37 \mathrm{MBq}$ and 18.5 MBq groups, respectively. Dose-dependent tumor growth suppression didn't observe. ${ }^{153} \mathrm{Sm}$-KARP-CheM didn't increase significantly average life span of treated animals. In conclusion, the suppression of tumor growth suggested that this compound has exciting potential for local tumor therapy.

\section{References}

[1] Vanparijs N., Nuhn L., De Geest B.G. Transiently thermoresponsive polymers and their applications in biomedicine. Chem. Soc. Rev., 2017, V. 46, No. 4, P. 1193-1239.

[2] Gandhi A., Paul A., Sen S.O., Sen K.K. Studies on thermoresponsive polymers: phase behaviour, drug delivery and biomedical applications. Asian J. Pharm. Sci., 2015, V. 10, P. 99-107.

[3] Haq M.A., Su Y., Wang D. Mechanical properties of PNIPAM based hydrogels: A review. Mater. Sci. Eng. C. Mater. Biol. Appl., 2017, V. 70(Pt 1), P. 842-855.

[4] Hruby M., Konak C., Kucka J., Vetrik M., Filippov S.K., Vetvicka D., Mackova H., Karlsson G., Edwards K., Rihova B., Ulbrich K. Thermoresponsive, hydrolytically degradable polymer micelles intended for radionuclide delivery. Macromol. Biosci., 2009, V. 9, P. 1016-1027.

[5] Kucka J., Hruby M., Lebeda O. Biodistribution of a radiolabelled thermoresponsive polymer in mice. Appl. Radiat. Isotopes., 2010, V. 68, P. 1073-1078. 